\title{
Lesões pigmentadas da mucosa oral: apresentação clínica, diagnóstico e tratamento
}

\author{
Pigmented lesions of the oral mucosa: clinical presentation, diagnosis and treatment
}

Lesiones pigmentadas de la mucosa oral: presentación clínica, diagnóstico y tratamiento

Marcela Nogueira dos Santos ORCID: https://orcid.org/0000-0002-0554-6382

Faculdade de Odontologia do Recife, Brasil E-mail: marcelaanogueira.santos@gmail.com

Nívia Delamoniky Lima Fernandes ORCID: https://orcid.org/0000-0001-8439-2117 Centro Universitário Inta, Brasil E-mail: delamonikynivia@gmail.com

Jefferson Douglas Lima Fernandes ORCID: https://orcid.org/0000-0002-5231-3813

Universidade Federal do Ceará, Brasil

E-mail: jefferson.odonto97@gmail.com

Jamille de Souza Castro

ORCID: https://orcid.org/0000-0003-3257-8651

Centro universitário Alfredo Nasser, Brasil E-mail: castrosjamille@gmail.com

Jaqueline Lopes Reis

ORCID: https://orcid.org/0000-0003-1685-0336

Universidade de Rio Verde, Brasil

E-mail: jaquelreis@hotmail.com

Bruna Peixoto Girard

ORCID: https://orcid.org/0000-0003-0233-666X

Centro Universitário Cesmac, Brasil

E-mail: brunapeixoto5@hotmail.com

Vitória Ribeiro Barbosa de Menezes

ORCID: https://orcid.org/0000-0001-6750-4770

Centro Universitário Maurício de Nassau, Brasil

E-mail: vickymenezes1999@gmail.com

Marcieli Borba do Nascimento

ORCID: https://orcid.org/0000-0002-8087-0801

Universidade Estadual do Centro-Oeste, Brasil

E-mail:marcieli_bn@hotmail.com

Hana Yasmim Marques Silva de Souza

ORCID: https://orcid.org/0000-0003-0128-7720

Centro Universitário Tiradentes de Pernambuco, Brasil

E-mail: hanayasmim@hotmail.com

Matheus Almeida Ramalho

ORCID: https://orcid.org/0000-0002-5607-1313

Universidade Federal de Jataí, Brasil

E-mail: matheusramalho@discente.ufg.br

Rayza Brito Silva

ORCID: https://orcid.org/0000-0003-2381-6398

Universidade Federal do Norte do Tocantins, Brasil

E-mail: rayza.silva180@gmail.com

Letícia Barbosa Gonçalves

ORCID: https://orcid.org/0000-0001-9377-2306

Universidade Federal do Ceará, Brasil

E-mail: leticiabarbosa@alu.ufc.br

Josiane Dias de Freitas Machado

ORCID: https://orcid.org/0000-0002-9548-8162 Centro Universitário Cesuca, Brasil

E-mail: jdjosimachado@gmail.com

Cleison da Silva Fernandes

ORCID: https://orcid.org/0000-0002-8427-699X Centro Universitário Unifg, Brasil E-mail: ferndscleei@gmail.com 


\begin{abstract}
Resumo
Lesões pigmentadas da mucosa oral compõem um grupo diverso de lesões que podem variar de pigmentações fisiológicas, como ocorre a pigmentação gengival em pessoas com pele negra, e pigmentações patológicas graves, como o melanoma oral. A identificação do tecido pigmentado na cavidade oral pode representar dificuldades quando ao diagnóstico para o profissional da saúde. Isso ocorre, pois, a manifestação clínica das lesões pigmentadas são diversificadas e pode variar de coloração macular focal a difusa ou, ainda, de um pequeno crescimento nodular a uma grande massa. Diante do exposto, esse estudo objetivou revisar a literatura acerca lesões pigmentadas da mucosa oral, destacando sua apresentação clínica, diagnóstico e tratamento, bem como a importância de um correto manejo clínico em pacientes que possuem tais lesões na cavidade oral. Para a construção deste trabalho foi feito um levantamento bibliográfico nas bases de dados SciVerse Scopus, Scientific Eletronic Library Online (Scielo), U.S. National Library of Medicine (PUBMED) e ScienceDirect, utilizando o gerenciador de referências Mendeley. Estudos científicos disponíveis na literatura relatam que há diversas lesões pigmentadas que acometem a mucosa oral. Essas complicações envolvem tatuagem por amálgama, mácula melanótica oral, nevo melanocítico, melanoma e melanoacantoma oral. Diante disso, é de extrema importância que os profissionais da saúde estejam familiarizados com essas lesões, distinguindo-as de lesões malignas que afetam a cavidade oral, a afim de que se estabeleça o correto tratamento para cada caso.
\end{abstract}

Palavras-chave: Lesões pigmentadas; Pigmentação oral; Etiologia; Tratamento; Diagnóstico.

\begin{abstract}
Pigmented lesions of the oral mucosa comprise a diverse group of lesions that can range from physiological pigmentations, such as gingival pigmentation in people with black skin, to severe pathological pigmentations, such as oral melanoma. The identification of pigmented tissue in the oral cavity can represent difficulties in the diagnosis for the health professional. This occurs because the clinical manifestations of pigmented lesions are diverse and can vary from focal to diffuse macular coloration, or even from a small nodular growth to a large mass. Given the above, this study aimed to review the literature on pigmented lesions of the oral mucosa, highlighting their clinical presentation, diagnosis and treatment, as well as the importance of corrective clinical management in patients who have such lesions in the oral cavity. For the construction of this work, a bibliographic survey was carried out in the SciVerse Scopus, Scientific Electronic Library Online (Scielo), U.S. National Library of Medicine (PUBMED) and ScienceDirect databases, using the Mendeley reference manager. Scientific studies available in the literature report that there are several pigmented lesions that affect the oral mucosa. These complications involve amalgam tattooing, oral melanotic macula, melanocytic nevus, melanoma, and oral melanoacanthoma. Therefore, it is extremely important that health professionals are familiar with these lesions, distinguishing them from malignant lesions that affect the oral cavity, in order to establish the correct treatment for each case.
\end{abstract}

Keywords: Pigmented lesions; Oral pigmentation; Etiology; Treatment; Diagnosis.

\title{
Resumen
}

Las lesiones pigmentadas de la mucosa oral comprenden un grupo diverso de lesiones que pueden ir desde pigmentaciones fisiológicas, como la pigmentación gingival en personas de piel negra, hasta pigmentaciones patológicas graves, como el melanoma oral. La identificación de tejido pigmentado en la cavidad bucal puede representar dificultades en el diagnóstico para el profesional de la salud. Esto ocurre porque las manifestaciones clínicas de las lesiones pigmentadas son diversas y pueden variar de coloración macular focal a difusa, o incluso de un pequeño crecimiento nodular a una gran masa. Dado lo anterior, este estudio tuvo como objetivo revisar la literatura sobre las lesiones pigmentadas de la mucosa oral, destacando su presentación clínica, diagnóstico y tratamiento, así como la importancia del manejo clínico correctivo en los pacientes que presentan dichas lesiones en la cavidad oral. Para la construcción de este trabajo se realizó un levantamiento bibliográfico en las bases de datos SciVerse Scopus, Scientific Electronic Library Online (Scielo), U.S. National Library of Medicine (PUBMED) y ScienceDirect, utilizando el gestor de referencias de Mendeley. Los estudios científicos disponibles en la literatura informan que existen varias lesiones pigmentadas que afectan la mucosa oral. Estas complicaciones incluyen tatuajes de amalgama, mácula melanótica oral, nevo melanocítico, melanoma y melanoacantoma oral. Por ello, es de suma importancia que los profesionales sanitarios conozcan estas lesiones, distinguiéndolas de las malignas que afectan a la cavidad bucal, para poder establecer el tratamiento correcto para cada caso.

Palabras clave: Lesiones pigmentadas; Pigmentación oral; Etiología; Tratamiento; Diagnóstico.

\section{Introdução}

Lesões pigmentadas da mucosa oral compõem um grupo diversos de lesões que podem variar de pigmentações fisiológicas, como ocorre a pigmentação gengival em pessoas com pele negra, e pigmentações patológicas graves, como o melanoma oral (Rosebush, Briody, and Cordell 2019). 
Essa pigmentação pode ser resultado do acúmulo de pigmentos endógenos e exógenos. Pigmentação exógena pode ser causada pelo aumento local de deposição de melanina e/ou aumento do número de melanócitos, além de aumento na produção de outros pigmentos como hemoglobina, hemossiderina e caroteno. Já a pigmentação exógena oral provém, como o próprio nome sugere, de um material exógeno, como a deposição de amálgama que dá origem às tatuagens por amálgama, além outros fatores que contribuem com o desenvolvimento da lesão como tabaco, uso de drogas, fumo ou indução de materiais pesados (Alawi 2013; Kumar, Kumar, and Agarwal 2014).

A identificação do tecido pigmentado na cavidade oral pode representar dificuldades quanto ao diagnóstico para o profissional da saúde. Isso ocorre, pois, a manifestação clínica das lesões pigmentadas são diversificadas e podem variar de coloração macular focal a difusa ou, ainda, de um pequeno crescimento nodular a uma grande massa (Neville 2009; Sreeja et al. 2015).

Diante do exposto, esse estudo objetivou revisar a literatura acerca lesões pigmentadas da mucosa oral, destacando sua apresentação clínica, diagnóstico e tratamento, bem como a importância de um correto manejo clínico em pacientes que possuem tais lesões na cavidade oral.

\section{Metodologia}

Trata-se de uma revisão narrativa da literatura. A revisão de literatura permite a busca aprofundada dentro de diversos autores e referenciais sobre um tema específico, nesse caso, as manifestações orais decorrentes da radioterapia de cabeça e pescoço (Pereira et al. 2018).

A fim de que haja direcionamento na pesquisa delineou-se como questão norteadora: "Quais são as lesões pigmentadas da mucosa oral, suas apresentações clínicas, diagnóstico e tratamento?"

Para a construção deste artigo foi realizado um levantamento bibliográfico nas bases de dados SciVerse Scopus, Scientific Eletronic Library Online (Scielo), U.S. National Library of Medicine (PUBMED) e ScienceDirect, com auxílio do gerenciador de referências Mendeley. Os artigos foram contemplados entre os anos de 2010 a 2021.

A estratégia de pesquisa desenvolvida para identificar os artigos incluídos e avaliados para este estudo baseou-se nos descritores contidos na lista dos Descritores em Ciência da Saúde (DeCS) e suas combinações no idioma português e inglês: [(lesões pigmentadas OR pigmented lesions) AND (mucosa oral OR oral mucosa OR boca OR mouth OR mucosa jugal OR cheek mucosa OR língua OR tongue) AND (manifestações clínicas OR clinical findings OR tratamento OR treatmente OR etiologia OR etiology OR diagnóstico OR diagnosis)].

\subsection{Critérios de inclusão e exclusão}

Considerou-se como critério de inclusão os artigos completos disponíveis na íntegra nas bases de dados citadas, nos idiomas inglês e português e relacionados com o objetivo deste estudo. Os critérios de exclusão foram artigos incompletos, duplicados, resenhas, estudos in vitro e resumos.

\subsection{Seleção de estudos}

A estratégia de pesquisa baseou-se na leitura dos títulos para encontrar estudos que investigassem a temática da pesquisa. Caso contemplasse esse primeiro objetivo, posteriormente, os resumos eram lidos e, persistindo na inclusão, era feita a leitura do artigo completo. Quando havia dúvida sobre a inclusão, o artigo era lido por outro autor e, a decisão de inclusão ou exclu são era tomada em consenso. 


\section{Resultados e discussão}

Com base na revisão de literatura feita nas bases de dados eletrônicas citadas, foram identificados 2795 artigos científicos, dos quais 324 estavam duplicados com dois ou mais índices. Após a leitura e análise do título e resumos dos demais artigos outros 2390 foram excluídos. Assim, 81 artigos foram lidos na integra e, com base nos critérios de inclusão e exclusão, apenas 22 artigos foram selecionados para compor este estudo. O fluxograma com detalhamento de todas as etapas de seleção está na Figura 1.

Figura 1 - Fluxograma de identificação e seleção dos estudos.

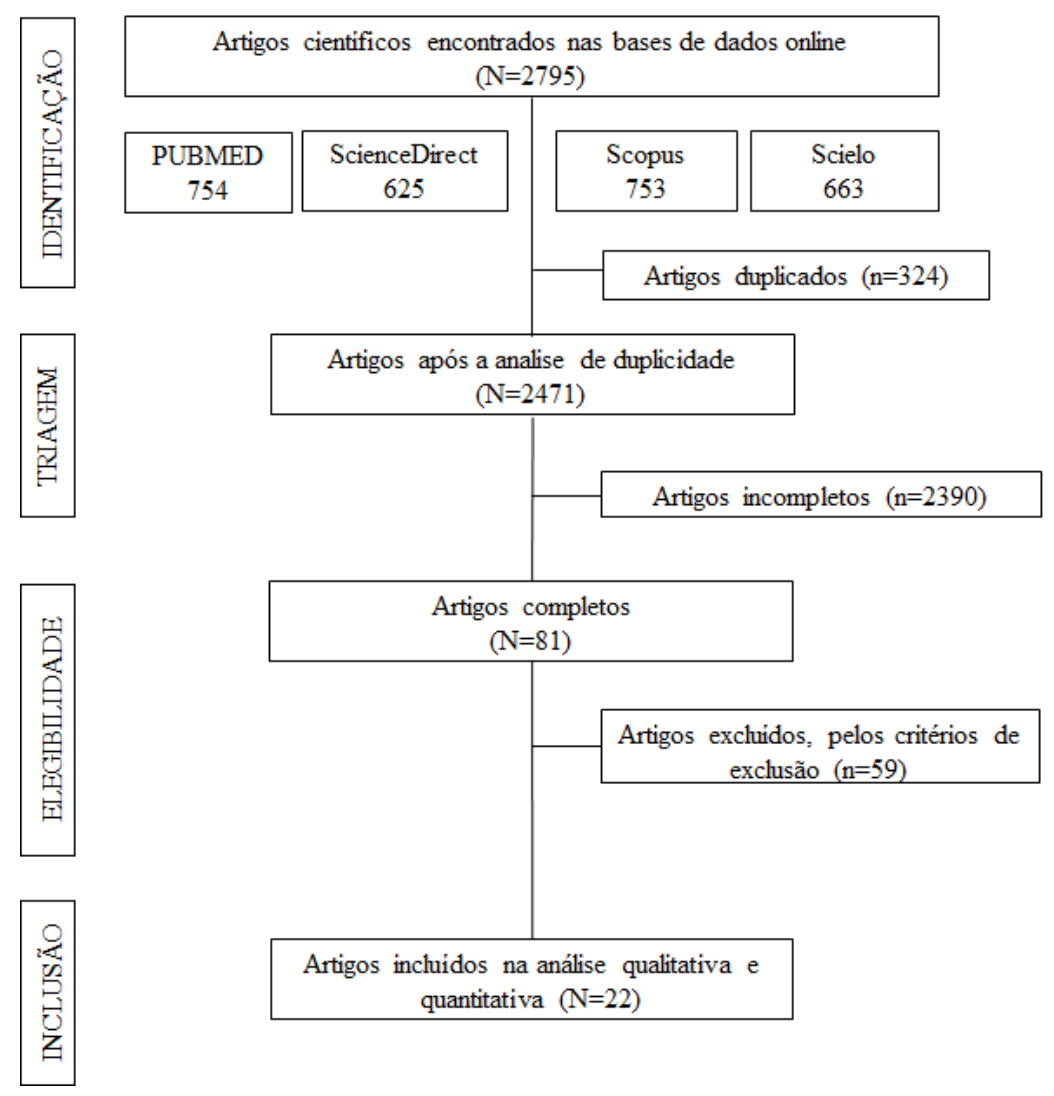

Fonte: Autores (2021).

Estudos científicos disponíveis na literatura relatam que há diversas lesões pigmentadas que acometem a mucosa oral. Essas complicações envolvem tatuagem por amálgama, mácula melanótica oral, nevo melanocítico, melanoma e melanoacantoma oral.

\subsection{Tatuagem por amálgama}

A pigmentação da mucosa bucal pode ocorrer devido à deposição de materiais estranhos exógenos, como o amálgama dentário, pigmento de tatuagem ou grafite. A tatuagem por amálgama (TA) é uma lesão iatrogênica, na qual o material pode ser incorporado nos tecidos moles por diversas formas (Neville 2009; Rosebush et al. 2019).

A TA pode ocorrer durante a colocação ou remoção de uma restauração, na qual os fragmentos do amálgama podem ser aderidos na mucosa bucal por abrasão, local de extração ou sulco gengival (Neville 2009; Rosebush et al. 2019).

Clinicamente, apresentam-se como manchas ou, raramente, como lesões ligeiramente elevadas, com uma coloração cinza, azul ou preta. As bordas podem ser bem definidas, irregulares ou difusas, normalmente com tamanho médio de 6 mm. O 
aumento pode ocorrer devido à migração de macrófagos, reabsorção do osso subjacente ou corrosão contínua. Embora qualquer superfície da mucosa possa estar envolvida, a gengiva, a mucosa alveolar e a mucosa jugal são os locais de maior recorrência para tatuagem por amálgama (Albuquerque et al. 2021; Neville 2009; Rosebush et al. 2019)

Com relação ao diagnóstico, o clínico pode realizar tomadas radiográficas das áreas de pigmentação, na tentativa de demonstrar os fragmentos metálicos. Em caso de dúvida, uma biopsia pode ser realizada, a fim de que seja estabelecido o diagnóstico final da lesão e excluir a possibilidade de neoplasia malanocítica (Kauzman, Blanas, and Frcd 2013; Neville 2009)

Não há necessidade de tratamento para as lesões por amálgama quando os fragmentos podem ser detectados radiograficamente. Em alguns casos, o local pigmentado pode resultar em comprometimento estético, como na região gengival ântero-superior. Nesses casos, a excisão cirúrgica conservadora pode ser realizada na área (Albuquerque et al. 2021; Neville 2009).

\subsection{Mácula Melanótica Oral}

É uma das lesões pigmentadas com maior recorrência que envolvem a cavidade oral. Normalmente apresentam-se como mácula redonda ou oval, solitárias, pequenas, bem circunscritas, de coloração uniforme variando de amarelo-amarronzado a marrom escuro, assintomáticas, com diâmetro de, no máximo 7 mm (Alawi 2013; Kumar et al. 2014; Neville 2009).

Ocorre com maior recorrência em mulheres com idade média de 43 anos. Embora qualquer local da cavidade oral possa ser acometido pela mácula melanótica, sabe-se que o lábio inferior, mucosa jugal, gengiva e palato são áreas mais acometidas (Alawi 2013; Kumar et al. 2014; Neville 2009).

A etiologia ainda não é bem estabelecida, porém sabe-se que traumas podem contribuir para a lesão. O diagnóstico da mácula melanótica é baseado em achados clínicos e, normalmente, uma biopsia é solicitada pelo clínico, a fim de descartar os diagnósticos diferenciais que exigem tratamento. Por se tratar de uma lesão sem complicações, a mácula melanótica não requer nenhum tratamento. Entretanto, como o local de maior recorrência é o lábio inferior, o paciente pode sugerir a remoção completa da mácula, por questões estéticas (Alawi 2013; Kumar et al. 2014)

Histologicamente é possível notar a pigmentação melânica abundante dentro da camada de células epiteliais basais com incontinência melânica nas porções superficiais da submucosa. Em casos de hiperplasia melanocítica deve-se levar em consideração o tratamento cirúrgico completa da lesão, devido ao seu potencial de malignização para o melanoma maligno (Alawi 2013; Kumar et al. 2014).

\subsection{Nevo melanocítico}

O nevo melanocítico é um grupo de tumores benignos resultantes do crescimento e proliferação dos melanócitos, sendo reconhecimentos como nevo epidérmico, nevo esponjoso branco, nevo sebáceo, nevo flâmeo, nevo de célula basal e outros. O nevo de maior recorrência é o nevo melanocítico adquirido (Neville 2009; Rosebush et al. 2019).

Acometem, majoritariamente, pessoas do sexo feminino com idade superior a trinta anos. Clinicamente, observa-se um nódulo ou mácula pequena, solitária, de coloração marrom ou azulada bem circunscrita e assintomática. Entretanto, há relato de nevos sem apresentação de pigmentação clínica. Qualquer local da mucosa bucal pode ser afetado, porém o palato duro, a mucosa bucal e labial e gengiva são os locais de maior recorrência (Neville 2009; Rosebush et al. 2019).

Em relação a etiologia, o nevo melanocítico pode ser congênito ou representar a manifestação de um distúrbio genético incomum. Mas, habitualmente, são lesões adquiridas, com fatores genéticos e ambientais desempenhando papel importante em seu desenvolvimento (Neville 2009; Rosebush et al. 2019).

O diagnóstico deve ser realizado por meio de achados clínicos e confirmado pelo exame histopatológico mediante biopsia, a fim de descartar o melanoma maligno. Algumas variantes do nevo melanocítico oral, como o nevo azul cutâneo, 
apresenta potencial para transformação maligna. Nesse caso, a cirurgia conservadora é o tratamento de melhor escolha para essas lesões orais (Neville 2009; Rosebush et al. 2019).

\subsection{Melanoma}

O melanoma é uma neoplasia maligna dos melanócitos epidérmicos da mucosa oral. A radiação ultravioleta tem sido considerada como fator causal para o desenvolvimento dessa neoplasia, uma vez que há maior predileção em pessoas da pele clara, quando comparada a pessoas de pele negra (Neville 2009; Sreeja et al. 2015)

Possuem quatro subtipos histológicos conhecido, sendo eles: melanoma de disseminação superficial, melanoma nodular, melanoma lentigo maligno e melanoma lentiginoso acral (Neville 2009; Sreeja et al. 2015).

O local de maior recorrência é o palato, com uma porcentagem de $40 \%$ dos casos, seguido pela gengiva, que corresponde a um terço dos casos. Acomete, majoritariamente pessoas do sexo masculino com idade superior a 50 anos (Neville 2009; Reis 2018; Sreeja et al. 2015).

Clinicamente, o melanoma manifesta-se como uma mancha assimétrica, de coloração enegrecida, irregular, variando entre o castanho ao negro, branco vermelho ou azul. O diâmetro é, normalmente, superior a 3 mm e os bordos. Alguns outros sinais e sintomas podem ser encontrados, como ulceração, dor, parestesia, mobilidade dentária ou esfoliação espontânea, reabsorção radicular e/ou perda óssea. Entretanto, em alguns casos, o paciente pode apresentar-se sem sintomatologia (Misır et al. 2016; Neville 2009; Reis 2018).

Por possui semelhanças clínicas semelhantes ao nevo melanocítico, um sistema de "ABCDE” foi proposto, como forma de avaliação e auxilio na distinção clinica entre o melanoma e o nevo melanocítico (Tabela 1).

Além dos achados clínicos e utilização do sistema "ABCDE", o diagnóstico do melanoma deve ser confirmado por meio de exames histopalotógico. Após a confirmação para melanoma oral, o tratamento cirúrgico deve ser realizado (Misır et al. 2016; Neville 2009).

Tabela 1 - Características clínicas que facilitam o diagnóstico do Melanoma.

\begin{tabular}{ll}
\hline \multicolumn{1}{c}{ Características Clínicas "ABCDE” para o melanoma } \\
\hline$\bullet$ & Assimetria (devido ao seu padrão de crescimento descontrolado) \\
- & Bordas irregulares (geralmente em serrilhado) \\
- & Coloração irregular (que varia de tons do marrom ao negro, branco, vermelho e azul, dependendo da \\
& quantidade e da profundidade da pigmentação por melanina) \\
$\bullet$ & Diâmetro maior do que 6 mm (que é o diâmetro de uma borracha de lápis) \\
- & Evolutivo (lesões que apresentam mudanças com relação ao tamanho, forma, cor, superfície ou \\
& sintomas ao longo do tempo)
\end{tabular}

Fonte: Neville (2009).

\subsection{Melanoacantoma oral}

O melanoacantoma oral é uma pigmentação adquirida e benigna, relativamente comum, marcada por melanócitos dendríticos dispersos por todo o epitélio. Apresenta-se como uma resposta reacional e, em alguns casos, o trauma e irritação crônica têm sido associados como fatores de ocorrência de tais lesões(Alawi 2013; Kumar et al. 2014; Neville 2009). 
É observado quase exclusivamente em pessoas negras, com predileção para o sexo feminino com idade entre 30 a 40 anos. A mucosa jugal é o local de maior recorrência, seguido por lábios, palato, gengiva e mucosa alveolar (Alawi 2013; Kumar et al. 2014; Neville 2009).

Clinicamente, o melanoacantoma oral apresenta-se como uma pigmentação macular, sem sintomatologia dolorosa, mal definida e de rápido crescimento, de superfície lisa, plana ou, em alguns casos, levemente elevada, com coloração de coloração marrom-escura a negra (Alawi 2013; Kumar et al. 2014; Neville 2009).

Devido à apresentação clínica, o melanoma é considerado no diagnóstico diferencial, tornando necessário a biopsia incisional no local. Uma vez que o diagnóstico histológico de melanoacantoma oral tenha sido estabelecido, nenhum tratamento adicional é necessário. O próprio procedimento de biópsia pode levar à regressão espontânea da lesão (Alawi 2013; Kumar et al. 2014; Lakshminarayanan and Ranganathan 2009; Müller 2010; Neville 2009).

\section{Considerações Finais}

Diante do exposto, esse estudo objetivou revisar a literatura acerca lesões pigmentadas da mucosa oral, destacando sua apresentação clínica, diagnóstico e tratamento, bem como a importância de um corretor manejo clínico em pacientes que possuem tais lesões na cavidade oral.

A pigmentação é definida como o processo de deposição de pigmentos nos tecidos. Várias doenças podem levar a colorações variadas na mucosa. Pode surgir de fatores intrínsecos e extrínsecos e pode ser fisiológico ou patológico. O dentista deve estar ciente das várias lesões para auxiliar no plano de tratamento adequado.

\section{Referências}

Alawi, F. 2013. "Pigmented Lesions of the Oral Cavity: An Update.” Dental Clinics of North America 57(4):699-710.

Albuquerque, D. M., J. L. Cunha, A. L. Roza, L. P. Arboleda, A. R. Santos-Silva, M. A. Lopes, P. A. Vargas, J. Jorge, O. P. de Almeida, A. C. Abrahão, M. Agostini, \& M. J. Romañach. 2021. "Oral Pigmented Lesions: A Retrospective Analysis from Brazil." Medicina Oral, Patologia Oral y Cirugia Bucal 26(3):e284-91.

Kauzman, A., \& Nick, B. C. Frcd. 2013. "Pigmented Lesions Of The Oral Cavity-Review And Differential Diagnosis." Chettinad Health City Medical Journal 2(2).

Kumar, M., Manish, K., \& Deshant, A. 2014. "Pigmented Lesion of Buccal Mucosa." Case Reports in Medicine 2014:936142.

Lakshminarayanan, V., \& Kannan, R. 2009. “Oral Melanoacanthoma: A Case Report and Review of the Literature.” Journal of Medical Case Reports 3:11.

Mısır, A. Ferhat, Mustafa C. Durmuşlar, Tamer Zerener, \& Banu D. Gün. 2016. “Primary Malignant Melanoma.” Saudi Medical Journal 37(4):446-49.

Müller, S. 2010. "Melanin-Associated Pigmented Lesions of the Oral Mucosa: Presentation, Differential Diagnosis, and Treatment." Dermatologic Therapy 23(3):220-29.

Neville, B. W; Douglas Damm; Carl A.; \& Jerry B. 2009. Oral and Maxillofacial Pathology. 3rd ed.

Pereira, A., Shitsuka, D.; Parreira, F. \& Shitsuka.; R.2018. Método Qualitativo, Quantitativo Ou Quali-Quanti.

Reis, R. O. 2018. "Diagnóstico Diferencial de Lesões Pigmentadas Da Cavidade Oral.”

Rosebush, M. S., Ashleigh N. Briody, \& Kitrina G. Cordell. 2019. "Black and Brown: Non-Neoplastic Pigmentation of the Oral Mucosa." Head and Neck Pathology 13(1):47-55.

Sreeja, C., K. Ramakrishnan, D. Vijayalakshmi, M. Devi, I. Aesha, \& B. Vijayabanu. 2015. "Oral Pigmentation: A Review.” Journal of Pharmacy \& Bioallied Sciences 7(Suppl 2):S403-8. 